УДК $512.64+512.55$

\title{
The Determinants over Associative Rings: a Definition, Properties, New Formulas and a Computational Complexity
}

\author{
Georgy P. Egorychev* \\ Institute of Mathematics and Computer Science \\ Siberian Federal University \\ Svobodny, 79, Krasnoyarsk, 660041
}

Russia

Received 17.06.2016, received in revised form 05.07.2016, accepted 15.09.2016

We give a new definition for the determinants over an associative ring $\mathbf{Q}$ and study their properties. In particular, we obtain a new family of polynomial identities (computational formulas) for these determinants that contain up to $n$ ! free variables.

Keywords: determinants, associative rings, noncommutative variables, the polarization theorem, polynomial identities.

DOI: $10.17516 / 1997-1397-2016-9-4-443-448$.

\section{Introduction}

Recently the author in $[1,2]$ has found a new family of formulas with free variables (polynomial identities) for the determinant over the commutative ring $K$. These formulas have alloved me to give a new definition for determinants (an e-determinant $\operatorname{edet}(A)$ ) over a wide class of rings $[1,3]$ and to establish their basic properties (without proof). These definitions (for a noncommutative case) are closely related to a definition of the symmetrized Barvinok determinant $\operatorname{sdet}(A)$ [4].

Here the complete proof of statements from [3] for $\operatorname{edet}(A)$ over the associative ring $\mathbf{Q}$ is given. With the help of knoun theorem of polarization $[5,6]$ to obtain a new family of polynomial identities for $\operatorname{edet}(A)$ that contain up to $n$ ! free variables. The basic propeties $\operatorname{edet}(A)$ are proved. These propeties coincide with many well-known propeties of a determinant over a commutative ring. The necessary and sufficient conditions of equality $\operatorname{edet}(A)=0$ are found, in particular. We also estimate a computational complexity of the obtained formulas for determinants. Some prospects of the basic results are specified.

\section{Notations and definitions}

Let $K$ be a commutative ring, $\mathbf{K}$ a noncommutative associative ring, $Q$ a noncommutative ring with associative $n$-powers (one-monomial associativeness), and $\mathbf{Q}$ be an associative ring; let also each ring be with division by integers. Let $A=\left(a_{i j}\right)$ be an $n \times n$ matrix with elements from the ring $K$. Let $S_{n}$ be the set of all permutations $\sigma=(\sigma(1), \ldots, \sigma(n))$ of the set $\{1, \ldots, n\}, \tau(\sigma)$ be the number of inversions in $\sigma$. Let $S_{n}^{(e)}$ and $S_{n}^{(o)}$ be the subsets of even and odd permutations in $S_{n}$, respectively. We call the sequence of elements of $a_{1 \sigma(1)}, \ldots, a_{n \sigma(n)}$ the diagonal $l(\sigma)$ of matrix $A$, the sequence of elements $a_{i_{1} \sigma\left(i_{1}\right)}, \ldots, a_{i_{k} \sigma\left(i_{k}\right)}, 1 \leqslant i_{1}<\ldots<i_{k} \leqslant n$, the subdiagonal $l$

*gegorych@mail.ru

(c) Siberian Federal University. All rights reserved 
of length $k$ of matrix $A$. We denote by $L_{k}^{(e)}\left(L_{k}^{(o)}\right)$ the set of all subdiagonals of length $k$ of для diagonals from $S_{n}^{(e)}\left(S_{n}^{(o)}\right), k=1, \ldots, n$.

Definition 1. A classic definition for the determinant over the commutative ring $K$ :

$$
\operatorname{det}(A):=\sum_{\sigma \in S_{n}}(-1)^{\tau(\sigma)} a_{1 \sigma(1)} \ldots a_{n \sigma(n)}=\frac{1}{n !} \sum_{\mu \in S_{n}} \sum_{\sigma \in S_{n}}(-1)^{\tau(\mu)+\tau(\sigma)} a_{\mu(1) \sigma(1)} \ldots a_{\mu(n) \sigma(n)} .
$$

One of natural generalizations of a determinant (1) on noncommutative rings has been made in 2000 by A. Barvinok who has successfully applied it for construction of an algorithm to calculate permanents [4]. This definition of determinant differs from the well-known determinant of Dieudonné over a division ring, the quasideterminant [7] and other well-known determinants over noncommutative associative rings [8].

Definition 2. The definition of symmetrized Barvinok determinant sdetA) over the ring $\mathbf{K}$, [4]:

$$
\begin{gathered}
\operatorname{sdet}(A):=\frac{1}{n !} \sum_{\mu \in S_{n}} \sum_{\sigma \in S_{n}}(-1)^{\tau(\mu)+\tau(\sigma)} a_{\mu(1) \sigma(1)} \ldots a_{\mu(n) \sigma(n)}= \\
=\sum_{\sigma \in S_{n}}(-1)^{\tau(\sigma)} \operatorname{Sym}\left(a_{1 \sigma(1)} \times a_{2 \sigma(2)} \ldots \times a_{n \sigma(n)}\right),
\end{gathered}
$$

then the symmetrization operator Sym on the product elements $x_{1}, x_{2}, \ldots, x_{n} \in \mathbf{K}$ is defined to be the following sum

$$
\operatorname{Sym}\left(x_{1} x_{2} \ldots x_{n}\right):=\frac{1}{n !} \sum_{\sigma \in S_{n}} x_{\sigma(1)} x_{\sigma(2)} \ldots x_{\sigma(n)} .
$$

The classical polarization theorem of recovering a polyadditive symmetric function from its values on a diagonal is fundamental in the algebraic theory of polynomials. This theorem is often applied in the theory of rings, the linear and polylinear algebra, the theory of functions, the theory of mixed volumes and in other areas of mathematics. We use it in the following simple and general enough form.

Theorem 1 (The polarization theorem, Egorychev, [6]). Let $H$ be the additive commutative semigroup $H^{(n)}=H \times \ldots \times H$. The function $f(x)=f\left(x_{1}, \ldots, x_{n}\right): H^{(n)} \rightarrow \mathbb{C}$, is symmetric and polyadditive if only if for any $\gamma$ from $H$ it admits the following representation (the polarization formula):

$$
\begin{gathered}
f\left(x_{1}, \ldots, x_{n}\right)=\left\{(-1)^{n} f(\gamma, \ldots, \gamma)+(-1)^{n-1} \sum_{i=1}^{n} f\left(\gamma+x_{i}, \ldots, \gamma+x_{i}\right)+\right. \\
+(-1)^{n-2} \sum_{i \prec j} f\left(\gamma+x_{i}+x_{j}, \ldots, \gamma+x_{i}+x_{j}\right)+\ldots+ \\
\left.+\sum_{1 \leqslant j_{1}<\ldots<j_{k} \leqslant n} f\left(\gamma+\sum_{l=1}^{k} x_{j_{l}}, \ldots, \gamma+\sum_{l=1}^{k} x_{j_{l}}\right)+\ldots+f\left(\gamma+\sum_{i=1}^{n} x_{i}, \ldots, \gamma+\sum_{i=1}^{n} x_{i}\right)\right\} / n !
\end{gathered}
$$

\section{Determinants over an associative ring}

We give new

Definition 3. The definition of e-determinant edet $(A)$ over the associative ring $\mathbf{Q}$ (Egorychev, [1], [3]):

$$
\operatorname{edet}(A):=\frac{1}{n !}\left\{\left(\sum_{l \in L_{n}^{(e)}} s u^{n}(l)-\sum_{l \in L_{n}^{(o)}} s u^{n}(l)\right)-\left(\sum_{l \in L_{n-1}^{(e)}} s u^{n}(l)-\sum_{l \in L_{n-1}^{(o)}} s u^{n}(l)\right)\right\},
$$


where the function $s u(l)$ on the sequence $l$ of elements $x_{1}, x_{2}, \ldots, x \in \mathbf{Q}$ is defined to be the sum of its elements.

In the following theorem we employ the polarization theorem to obtain a new family of polynomial identities for $\operatorname{edet}(A)$ that contain up to $n$ ! free variables.

Theorem 2 ([3]). If $A=\left(a_{i j}\right)$ is an $n \times n$ matrix over the ring $\mathbf{Q}$, then the folloving formulas are valid

$$
\operatorname{edet}(A)=\frac{1}{n !} \sum_{\sigma \in S_{n}}(-1)^{\tau(\sigma)}\left\{(-1)^{n} \gamma_{\sigma}^{n}+\sum_{k=1}^{n}\left((-1)^{n-k} \sum_{1 \leqslant j_{1}<\ldots<j_{k} \leqslant n}\left(\gamma_{\sigma}+\sum_{s=1}^{k} a_{j_{s} \sigma\left(j_{s}\right)}\right)^{n}\right)\right\}
$$

where $\left\{\gamma_{\sigma}\right\}_{\sigma \in S_{n}}$ is the set of $n$ ! free variables $\gamma_{\sigma} \in \mathbf{Q}$.

Proof. If the ring $\mathbf{Q}$ is commutative then the validity of the formula (7) has been established by the author in [2]. If $\mathbf{Q}=\mathbf{K}$ then the formula (7) for $\operatorname{edet}(A)$ has been mentioned (without proof) in [3]. Its validity directly follows from several simple statements of Lemmas 3-5.

Lemma 3. The formula (7) is valid for the determinant sdet(A) over the ring $\mathbf{K}$ in (3).

Proof. As the symmetrization operator (4) is a symmetric and polyadditive function of its variables, then by means of the polarization formula for each diagonal product $a_{1 \sigma(1)} \ldots a_{n \sigma(n)}$, $\sigma \in S_{n}$, of elements from $\mathbf{K}$ we obtain

$$
\begin{aligned}
\operatorname{Sym}\left(a_{1 \sigma(1)} \times a_{2 \sigma(2)} \ldots \times a_{n \sigma(n)}\right) & = \\
= & \frac{1}{n !}\left\{(-1)^{n} \gamma_{\sigma}^{n}+\sum_{k=1}^{n}\left((-1)^{n-k} \sum_{1 \leqslant j_{1}<\ldots<j_{k} \leqslant n}\left(\gamma_{\sigma}+\sum_{s=1}^{k} a_{j_{s} \sigma\left(j_{s}\right)}\right)^{n}\right)\right\},
\end{aligned}
$$

where $\gamma_{\sigma}$ is an arbitrary variable from $\mathbf{K}$. To complete the proof it is enough to replace under the sign of the sum (3) each summand by (8).

Lemma 4. The following formula is valid for the determinant $\operatorname{sdet}(A)$ over the ring $\mathbf{K}$ :

$$
\begin{aligned}
\operatorname{sdet}(A)=\frac{1}{n !}\left\{\left(\sum_{l \in L_{n}^{(e)}}(\gamma+s u(l))^{n}-\right.\right. & \left.\sum_{l \in L_{n}^{(o)}}(\gamma+s u(l))^{n}\right)- \\
& \left.-\left(\sum_{l \in L_{n-1}^{(e)}}(\gamma+s u(l))^{n}-\sum_{l \in L_{n-1}^{(o)}}(\gamma+s u(l))^{n}\right)\right\},
\end{aligned}
$$

fo any $\gamma \in \mathbf{K}$. In particular for $\gamma=0$

$$
\operatorname{sdet}(A)=\frac{1}{n !}\left\{\left(\sum_{l \in L_{n}^{(e)}} s u^{n}(l)-\sum_{l \in L_{n}^{(o)}} s u^{n}(l)\right)-\left(\sum_{l \in L_{n-1}^{(e)}} s u^{n}(l)-\sum_{l \in L_{n-1}^{(o)}} s u^{n}(l)\right)\right\} .
$$

Proof. Put in (7) $\gamma_{\sigma}=\gamma \in \mathbf{K}$ for each $\sigma \in S_{n}$. Then we have

$$
\begin{aligned}
& \operatorname{sdet}(A)=\frac{1}{n !} \sum_{\sigma \in S_{n}}(-1)^{\tau(\sigma)}\left\{(-1)^{n} \gamma^{n}+\sum_{k=1}^{n}\left((-1)^{n-k} \sum_{1 \leqslant j_{1}<\ldots<j_{k} \leqslant n}\left(\gamma+\sum_{s=1}^{k} a_{j_{s} \sigma\left(j_{s}\right)}\right)^{n}\right)\right\}= \\
& =\frac{(-1)^{n}}{n !} \sum_{\sigma \in S_{n}}(-1)^{\tau(\sigma)} \gamma^{n}+\frac{1}{n !} \sum_{k=1}^{n}(-1)^{n-k}\left\{\sum_{\sigma \in S_{n}}(-1)^{\tau(\sigma)} \sum_{1 \leqslant j_{1}<\ldots<j_{k} \leqslant n}\left(\gamma+\sum_{s=1}^{k} a_{j_{s}} \sigma\left(j_{s}\right)\right)^{n}\right\}=
\end{aligned}
$$




$$
\begin{array}{r}
=\frac{(-1)^{n}}{n !}\left\{\sum_{\sigma \in S_{n}^{(e)}} \gamma^{n}-\sum_{\sigma \in S_{n}^{(o)}} \gamma^{n}\right\}+\frac{1}{n !} \sum_{k=1}^{n}(-1)^{n-k}\left\{\sum_{\sigma \in S_{n}^{(e)}} \sum_{1 \leqslant j_{1}<\ldots<j_{k} \leqslant n}\left(\gamma+\sum_{s=1}^{k} a_{j_{s} \sigma\left(j_{s}\right)}\right)^{n}-\right. \\
\left.\quad-\sum_{\sigma \in S_{n}^{(e)}} \sum_{1 \leqslant j_{1}<\ldots<j_{k} \leqslant n}\left(\gamma+\sum_{s=1}^{k} a_{j_{s} \sigma\left(j_{s}\right)}\right)^{n}\right\} .
\end{array}
$$

It is easy to see that the first term in the right part of (11) is equal to zero, as for any $n \geqslant 2$, obviosly $\left|S_{n}^{(e)}\right|=\left|S_{n}^{(o)}\right|$. Similarly, if we fix any $k, k=1, \ldots, n-2$ and any set elements $a_{j_{1} \sigma\left(j_{1}\right)}, a_{j_{2} \sigma\left(j_{2}\right)}, \ldots, a_{j_{s} \sigma\left(j_{s}\right)}$ of matrix $A$ then, obviously, this set of elements contains exactly $(n-k) ! / 2$ even and $(n-k) ! / 2$ odd permutations from the set $S_{n}$. Thus, under these assumptions any term $\left(\gamma+\sum_{s=1}^{k} a_{j_{s} \sigma\left(j_{s}\right)}\right)^{n}$ appears in the sum in the right part of the equality (11) the equal number of times with different signs, and therefore their sum is equal to zero. The remaining terms at $k=n-1$ and $k=n$ coincide with the expression in the right part of the equality (9) for $\operatorname{sdet}(A)$.

The following assertion follows from the equality of expressions (6) and (10) for $\operatorname{edet}(A)$ and $\operatorname{det}(A)$.

Lemma 5. The following formula is valid for the determinant edet $(A)$ over the ring $\mathbf{K}$ :

$$
\operatorname{edet}(A)=\operatorname{det}(A)
$$

Therefore for edet $(A)$ over $\mathbf{K}$ the formula (7) and its corollaries are valid.

Theorem 6 ([3]). The propeties edet $(A)$ over the associative ring $\mathbf{Q}$ :

(a) $\operatorname{edet}(A)$ is a polyadditive function of rows and columns of matrix A;

(b) $\operatorname{edet}(A)$ ecms is an antisymmetric function of rows and columns of matrix A;

(c) $\operatorname{edet}(A)=0$, if the matrix $A$ contains zero row or zero column;

(d) $\operatorname{edet}(A)=0$, if the matrix $A$ contains two equal rows (columns);

(e) $\operatorname{edet}(A)=\operatorname{edet}\left(A^{T}\right)$;

$(f)$ edet $\left(I_{n}\right)=e$, if the ring $\mathbf{Q}$ contains the unit $e$, and $I_{n}$ is the identity $n \times n$ matrix. valid.

In general case the Laplace formulas and the formula edet $(A B)=\operatorname{edet}(A) \cdot \operatorname{edet}(B)$ are not

Proof. If the ring $\mathbf{Q}$ is commutative, then the validity of the properties $(a)-(f)$ has been established by the author in [2]. If $\mathbf{Q}=\mathbf{K}$, then we use the equality $\operatorname{edet}(A)=\operatorname{sdet}(A)$ over $\mathbf{K}$ (Lemma 5).The rest of the proof of these properties goes along the well-known scheme for the determinant over the commutative ring $K$.

Corollary 7. If $A=\left(a_{i j}\right)$ is an $n \times n$ matrix over $\mathbf{Q}$ then for $t=1,2, \ldots, n-1$ following identities are valid:

$$
\sum_{l \in L_{n}^{(e)}} s u^{t}(l)-\sum_{l \in L_{n}^{(o)}} s u^{t}(l)=\sum_{l \in L_{n-1}^{(e)}} s u^{t}(l)-\sum_{l \in L_{n-1}^{(o)}} s u^{t}(l)
$$

and $\operatorname{edet}(A)=0$ iff the following identity holds:

$$
\sum_{l \in L_{n}^{(e)}} s u^{n}(l)-\sum_{l \in L_{n}^{(o)}} s u^{n}(l)=\sum_{l \in L_{n-1}^{(e)}} s u^{n}(l)-\sum_{l \in L_{n-1}^{(o)}} s u^{n}(l) .
$$


The coefficients at each power $\gamma^{t}, t=1, \ldots, n$ of the polynomial in the right part of (9) are equal to zero, as coefficients of this polynomial identity with respect to a free variable $\gamma$. Now to prove (13) it is enough to count up these coefficients by differentiation by $\gamma$ of the right part of (13) or, equivalently, using the binomial formula (with noncommutative variables) for each term in the right part of $(9)$.

Remark 8. In [2] it is proved, that the equality (14) can be considered as a (new) criterion for linear independence of rows (columns) of the matrix $A$ over the commutative ring $K$. If the rows (the columns) of the matrix $A$ over the noncommutative ring $\mathbf{K}$ are linearly dependent, then due to theorem $2 \operatorname{edet}(A)=0$, i. e. equality (14) holds. However, it is obvious, that the converse is not always true.

Lemma 9 (The computational complexity of $\operatorname{edet}(A)$ over the $\operatorname{ring} \mathbf{K})$. The computation edet $(A)$ by formulas (7) and (10) for large $n$ requires (at most) of order $(2 n-1) \times(n+1)$ ! additions and $(n+1) ! \times \ln n$ multiplications.

Proof. In [9] an (optimal) algorithm is given that allows for large $n$ to compute $2^{n}$ (starting with 2) by $\ln n$ operations of multiplication. In turn, it is easy to count thet the sum (7) contains only $(n+1)$ ! summands ( $n$-powers).

Remark 10. It is clear that the sum (2) for sdet $(A)$ contains $(n !)^{2}$ terms. Thus the traditional approach of computation sdet $(A)$, that uses computation of each term of (determinant) sum (2) requares of order $(2 n-1) \times(n !)^{2}$ additions and $(n-1) \times(n !)^{2}$ multiplications.

\section{Conclusion}

Here we make several short remarks explaining the basic results of this article and some prospects of their use.

Remark 11. (a) The formulas (7)-(14) are obtained using all characteristic properties of the e-determinant (compare [10]);

(b) Formula (7) for $n$ ! free variables $\left\{\gamma_{\sigma}\right\}_{\sigma \in S_{n}}$ generates $2^{n !}$ various polynomial identities if we put each $\gamma_{\sigma}=0$ or $\gamma_{\sigma} \neq 0$. Each of these formulas can be taken as the definition for $\operatorname{edet}(A)$, it requries its own number of arithmetical operations when calculated and according to Corollary 7 generates a new set of identities;

(c) Formula (7) and its special cases employ (besides divisions by $n$ !) only operations,+- and raising to power $n$. Therefore the formula (7) can be used as a definition of the e-determinats over noncommutative ring $Q$ with associative n-powers, keeping the properties $(a)-(f)$ of Theorem 6 .

It is of interest to obtain similar results for Shur functions, the mixed discriminants and many other matrix functions of planar and space matrices related to determinants. Our results may find applications in the theory of permanents [11], the tensor algebra and its applications, the theory of $n$-Lie algebras [12,13] and the theory of associative rings [14] and of noncommutative skew fields [15], the differential geometry [16] and others.

The author is grateful to his colleagues P.S. Kolesnikov, V. M. Kopytov, V. P. Krivokolesko, V. M. Levchuk, A. A. Makhnev, Y. N. Nuzhin, I. P. Shestakov, A. V. Shchuplev, for discussion of the basic results of this work and a number of useful remarks.

The research supported by the grant RFFI the project 16-01-00\%0\%.

\section{References}

[1] G.P.Egorychev, A new family of polynomial identities for computing determinants, Dokl. Math., 88(2013), no. 2, 510-512. 
[2] G.P.Egorychev, New polynomial identities for determinants over commutative rings, Izvestia Irkutsk. Gos. Univ., Seriya Matematika, 5(2012), no. 4, 16-20 (in Russian).

[3] G.P.Egorychev, A new family of polynomial identities for computing determinants, Dokl. Akad. Nauk, 452(2013), no. 1, 1-3 (in Russian).

[4] A.Barvinok, New permanet estimators via non-commutative determinants, Arxiv preprint math/0007153, 2000.

[5] H.Cartan, Elementary theory of analytic functions of one or several complex variables, Dover Publ., New York, 1995.

[6] G.P.Egorychev, New formulas for the permanent, Soviet Math. Dokl, 254(1980), no. 4, 784-787.

[7] I. M.Gel'fand, V.S.Retakh, Determinants of matrices over noncommutative rings, Funktsional. Anal. i Prilozhen., 25(1991), no. 2, 13-25 (in Russian).

[8] V.Arvind, S.Srinivasa, On the Hardness of Noncommutative Daterminant, Electronic Colloquium on Computational Camplexity, 103(2009), 1-18.

[9] V.V.Kochergin, About complexcity of computation one-terms of powers, Diskretnyi Analiz i Issledovanie Operatsii, 27(1994), 94-107 (in Russian).

[10] T.A.Muir, Treatise on the Theory of Determinants, Dover Publ., New York, 1960.

[11] G.P.Egorychev, Discrete mathematics. Permanents, Krasnoyarsk, Siberian Federal University, 2007.

[12] V.T.Filippov, On the n-Lie algebra of Jacobians, Sibirsk. Mat. Zh., 39(1998), no. 3, 660-669 (in Russian).

[13] A.P.Pozhidaev, A simple factor-algebras and subalgebras of Jajkobians algebras, Sibirsk. Mat. Zh., 39(1998), no. 3, 593-599 (in Russian).

[14] K.A.Zevlakov, A.M.Slin'ko, I.P.Shestakov, A.I.Shirshov, Rings that are nearly associative, Acad. Press, 1982.

[15] P.S.Kolesnikov, About different definitions of algebraically closed skew fields, Algebra and Logic, 40(2001), no. 4, 396-414.

[16] L.V.Sabinin, Methods of Nonassociative Algebra in Differential Geometry. In Supplement to Russian translation of S.K. Kobayashi and K. Nomizu, Foundations of Differential Geometry, 1, Moscow, Nauka, 1981 (in Russian).

\section{Детерминанты над ассоциативными кольцами: определение, свойства, новые формулы и сложность вычисления}

\section{Георгий П. Егорычев}

Дано новое определение детерминантов над ассочиативным кольцом $\mathbf{Q}$, и изучень их свойства. В частности, для них получено семейство полиномиальных тождеств (формул вычисления), содержащих до $n$ ! свободных параметров.

Ключевые слова: детеминанты, ассоциативные колъца, некоммутативные переменные, теорема поляризачии, полиномиалъные тождества. 